\title{
PREVALÊNCIA DE OBESIDADE INFANTIL NA ATENÇÃO PRIMÁRIA EM UMA CIDADE DO ALTO SERTÃO NO BRASIL
}

\author{
PREVALENCE OF CHILDHOOD OBESITY IN PRIMARY ATTENTION \\ IN A CITY OF THE HIGH SERTÃO IN BRAZIL
}

\author{
Raimundo Nonato da Costa Neto ${ }^{1}$ \\ José Alex Carneiro de Sousa ${ }^{2}$ \\ Ankilma do Nascimento Andrade Feitosa ${ }^{3}$ \\ Osvaldo Rui Dias Martins Filho ${ }^{4}$
}

\begin{abstract}
RESUMO: Introdução: A obesidade infantil é uma doença que apresenta uma série de prejuízos para a saúde das crianças, as quais são mais propensas a desenvolver futuramente doenças cardiovasculares, como hipertensão, acidente vascular cerebral, diabetes e dislipidemia. Observa-se que há um aumento da sua prevalência no Brasil e em outros países do mundo. Os dados mostram uma alta presença dessa doença, chegando a uma taxa de 10\% entre crianças de 5 a 9 anos de idade. Alguns fatores aumentam as chances do aparecimento de obesidade na infância, como a obesidade materna durante a gestação, o baixo nível socioeconômico e a baixa escolaridade materna. Dessa forma, por ser uma doença com sérias consequências, deve-se haver uma maior atenção voltada para identificação e intervenção precoces a fim de reduzir a sua prevalência e as suas complicações clínicas. Objetivo: Verificar a prevalência de obesidade infantil na atenção primária em uma cidade do alto sertão no Brasil. Metodologia: Tratou-se de um estudo de campo de caráter exploratório, descritivo, com abordagem quantitativa. A pesquisa foi realizada em 5 UBS's da cidade de Cajazeiras-PB, a
\end{abstract}

\footnotetext{
${ }_{1}^{1}$ Autor. Acadêmico do Curso de Graduação em Medicina da Faculdade Santa Maria, Cajazeiras - PB. Email: raimundoneto210@gmail.com.

2 Graduado em Medicina pela Universidade Federal de Campina Grande (2010). Residências em Clinica Médica (2011-2013) e Endocrinologia e Metabologia (2013-2015) pela UFCG, no Hospital Universitário Alcides Carneiro. Pós graduação em Medicina Intensiva pela Faculdade Redentor / AMIB.

${ }^{3}$ Doutora em Ciências da Saúde pela Faculdade de Medicina do ABC. Possui Mestrado (2010) e Licenciatura (2009) em Enfermagem Pela Universidade Federal da Paraíba, Especialização em Auditoria em Serviços de Saúde e Especialização em Saúde da Família pela UFPB, Graduação em Enfermagem pela Faculdade Santa Emília de Rodat (2005). Email: ankilmar@hotmail.com.

${ }^{4}$ Graduado em Medicina pela Faculdade de Ciências Médicas, Paraíba. Especializado em Radiologia Médica e Pós graduando em Medicina do Trabalho. Associado ao Colégio Brasileiro de Radiologia e Diagnóstico por Imagem, Membro da Sociedade Paulista de Radiologia, Membro da American Roentgen Ray Society.
} 
qual conta com 17 UBS's urbanas. A população foi de crianças na faixa etária de 2 a 11 anos que estavam sendo acompanhadas pela puericultura e realizando suas medidas antropométricas no dia do peso. A coleta de dados foi realizada com um questionário semiestruturado com parâmetros, como peso, idade, sexo e altura, para a caracterização das crianças e foi feita entre os meses de novembro de 2017 e fevereiro de 2018. A amostra foi composta por 98 crianças com idade entre 2 e 11 anos. Resultados e Discussão: A prevalência de crianças com sobrepeso foi de $12,2 \%$ e de crianças com obesidade foi de $25,5 \%$, confirmando, dessa forma, uma alta taxa de participantes com excesso de peso. Tais dados são bastante preocupantes, pois a obesidade é uma doença que apresenta sérias consequências para a saúde, diminuindo a qualidade de vida dos seus portadores. Entre as crianças com sobrepeso e obesidade, $32,4 \%$ das mães ainda não tinham concluído o ensino fundamental. Muitos estudos analisados conseguiram apontar relação entre - grau de escolaridade da mãe e presença de obesidade em seus filhos. Conclusão: Por meio da análise, percebe-se que houve a caracterização das crianças quanto ao grau de obesidade e sobrepeso e sua prevalência. Alguns fatores sociodemográficos foram relacionados à ocorrência de obesidade na infância, como a baixa escolaridade materna. Assim, espera-se que essa pesquisa contribua para alertar as pessoas que essa doença vem progredindo de forma preocupante na sociedade e se não evitada de forma adequada, pode causar sérias consequências para a saúde da população infantil.

Descritores: Atenção Primária; Obesidade Infantil; Prevalência; Puericultura.

ABSTRACT: Introduction: Childhood obesity is a disease that presents a number of health damage to children, who are more likely to develop cardiovascular diseases in the future, such as hypertension, cerebrovascular accident, diabetes and dyslipidemia. Its prevalence has increased in Brazil and in other countries of the world. Data show a high presence of this disease, reaching a rate of $10 \%$ among children from 5 to 9 years old. Some factors increase the chances of childhood obesity, such as maternal obesity during pregnancy, low socioeconomic status and low maternal schooling. Therefore, since it is a disease with serious complications, there should be greater attention at early identification and intervention in order to reduce its prevalence and clinical complications. Objective: To verify the prevalence of childhood obesity in primary care in a city in the Brazilian high backwoods region. Methodology: This was an exploratory, descriptive field study with a quantitative approach. The research was carried out in 5 UBS's of the city of Cajazeiras-PB, which has 17 UBS's urban. The population consisted of children in the age group from 2 to 11 years who were being accompanied by the puericulture and performing their anthropometric measures in the day of the weight. Data collection was performed with a semi-structured questionnaire with parameters such as weight, age, sex and height, for the characterization of the children and was done between November 2017 and February 2018. The sample consisted of 98 children with age between 2 and 11 years. Results and Discussion: The prevalence of overweight children was $12.2 \%$ and of children with obesity was $25.5 \%$, thus confirming a high rate of overweight participants. Such data are very worrying, since obesity is a disease that presents serious consequences for health, reducing the quality of life of 
its patients. Among overweight and obese children, 32.4\% of the mothers had not yet completed elementary school. Many studies have been able to point out the relation between the mother's degree of schooling and the presence of obesity in her children. Conclusion: Through the analysis, it was noticed that the children were characterized as to the degree of obesity and overweight and its prevalence. Some sociodemographic factors were related to the occurrence of childhood obesity, such as low maternal schooling. Thus, this research is expected to help to alert people that this disease is progressing in a worrying way in society and, if not avoided appropriately, it can bring serious consequences for the health of the child population.

Keywords: Primary attention; Child obesity; Prevalence; Childcare. 


\section{INTRODUÇÃO}

A obesidade é um problema que envolve alterações em níveis fisiológicos, metabólicos, anatômicos e psicossociais do organismo humano, cuja principal característica é uma elevação no peso corporal e no acúmulo de gorduras nos tecidos. Os estudos relatam que há fatores genéticos envolvidos nessa patologia, os quais se relacionam diretamente com os fatores ambientais, como dieta e sedentarismo. Entre as causas da obesidade, relata-se que uma ingestão calórica elevada associada com uma diminuição nas práticas de exercícios físicos configuram-se como seus fatores determinantes (SIMON et al., 2009).

A obesidade infantil está crescendo de forma assustadora nas últimas décadas, sendo considerada um problema de saúde pública. Ela pode trazer prejuízos em curto e em longo prazo para a saúde das crianças, as quais podem sofrer de apneia do sono mais facilmente e também passar por problemas sociais e psicológicos, como baixa autoestima e depressão. Crianças obesas representam um grupo de risco para doenças cardiovasculares como hipertensão e acidente vascular cerebral e são mais propensas a desenvolver diabetes mellitus e dislipidemia (JORTBERG et al., 2016).

No Brasil, observa-se um aumento da prevalência da obesidade infantil. Os dados mostram uma alta presença dessa doença, chegando a uma taxa de 10\% entre crianças de 5 a 9 anos de idade. De acordo com o Programa Nacional de Demografia e Saúde (PNDS) de 2006, 7\% das crianças brasileiras com idade inferior a 5 anos encontram-se com um nível excedente de peso. Segundo dados de 2009 da Associação Brasileira para o Estudo da Obesidade e da Síndrome Metabólica (ABESO), o excesso de peso em crianças entre 5 e 9 anos na região Nordeste encontra-se por volta de 28,15\% (BARBOSA et al., 2014; SIMON et al., 2009; POF, 2009).

Existem fatores que aumentam as chances do surgimento de obesidade na infância, dentre eles, pode-se citar a obesidade materna durante a gestação, o 
elevado peso ao nascer, o baixo nível socioeconômico e a baixa escolaridade materna. Os estudos demonstram que a origem da obesidade na infância é de caráter multifatorial, porém apresentando uma prevalência dos fatores exógenos, como uma alimentação inadequada e a falta de exercícios físicos $(\mathrm{SCHUCH}, 2012$; RODRIGUES; ALVES e AMORIM, 2014).

A identificação precoce, o diagnóstico e o acompanhamento clínico adequado da obesidade infantil são, portanto, importantes ações para reduzir a carga de doenças crônicas e deficiência na vida adulta. Os profissionais da saúde da atenção primária desempenham um papel importante no diagnóstico, educação e tratamento de crianças que são obesas já que geralmente constituem o primeiro ponto de contato dentro do sistema de saúde (KUHLE et al., 2015).

Diante da relevância da atenção primária em saúde no diagnóstico inicial e no acompanhamento clínico de seus usuários, levantou-se a ideia de pesquisar mais sobre a prevalência de obesidade infantil nas Unidades Básicas de Saúde do Alto Sertão Paraibano, já que essa doença vem crescendo de forma assustadora na atualidade.

\section{METODOLOGIA}

Tratou-se de uma pesquisa de campo de natureza exploratória e descritiva com abordagem quantitativa.

A pesquisa foi realizada em cinco Unidades de Saúde da Atenção Primária, localizadas na Zona Urbana da cidade de Cajazeiras-PB, a qual fica na mesorregião do Sertão Paraibano.

O nível de confiança desejado para esta pesquisa foi de $95 \%$, e um erro amostral $e=5 \%$. Com o resultado do cálculo amostral, o $n$ foi igual a 340 , sendo importante considerar que o número de crianças na faixa etária pré-estabelecida é variável. Dentre os critérios de inclusão, podem-se citar as crianças dentro da faixa etária de 2 a 11 anos que procuram as UBS's com seus pais e participam da puericultura. Os critérios de exclusão foram para as crianças que não estão na faixa 
etária já mencionada e também as que apresentam alguma comorbidade que interfira no seu peso.

Após a aplicação dos critérios de inclusão e exclusão propostos pelo estudo, chegou-se a um total de 98 crianças, pois a maioria das Unidades de Saúde da cidade realiza o dia do peso com crianças abaixo de dois anos e algumas crianças não foram acompanhadas pelos seus pais, mas por outros parentes.

O projeto de pesquisa foi encaminhado para o Comitê de Ética em Pesquisa (CEP) da Faculdade Santa Maria, localizado no município de Cajazeiras - PB, para apreciação. Depois de deferido o pedido, e de posse do parecer № 2.386.563, autorizando o início dos trabalhos, iniciou-se a coleta dos dados.

A coleta de dados foi realizada com um questionário semiestruturado com parâmetros e questões para a caracterização das crianças e dados pertinentes aos objetivos da pesquisa. No instrumento, foram utilizadas as seguintes variáveis: idade da criança; sexo; peso, altura e o cálculo do Índice de Massa Corporal (IMC); condições socioeconômicas como renda familiar e nível de escolaridade dos pais. As questões norteadoras do questionário foram elaboradas pelo pesquisador participante e pelo seu orientador.

Os dados foram coletados no período de novembro de 2017 a fevereiro de 2018 durante os dias que funcionam a puericultura ou que são feitas as medidas do peso e da altura pelos agentes comunitários de saúde. Antes da aplicação, os pais foram informados sobre os objetivos e a importância do projeto e assinaram o Termo de Consentimento Livre e Esclarecido (TCLE) juntamente com o Termo de Assentimento do Menor. Os participantes foram esclarecidos sobre quaisquer aspectos, tais como: o objetivo da pesquisa, o procedimento, a contribuição da pesquisa, a liberdade de se negar a participar do estudo.

Os dados foram analisados no SPSS (versão 25). Além de estatísticas descritivas de tendência central (média e mediana) e de dispersão (desvio padrão valores mínimos e máximos), também realizaram-se estatísticas inferenciais não paramétricas H de Kruskal-Wallis e teste de Qui-quadrado de Pearson. Foi aceito um critério de significância estatística menor ou igual a $5 \%$, ou seja, $p \leq 0,05$. 


\section{RESULTADOS E DISCUSSÃO}

O presente estudo foi realizado por meio da coleta de dados das crianças que frequentam a Estratégia Saúde da Família na cidade de Cajazeiras na Paraíba. A pesquisa consistiu em realizar a caracterização e analisar os dados de cada paciente. A amostra foi composta por 98 crianças com idade entre 02 e 11 anos.

No que diz respeito ao perfil das crianças, observou-se que a maioria era do sexo masculino, sendo 59 meninos $(60,2 \%)$ e 39 meninas $(39,8 \%)$. Em relação à idade, foi predominante a faixa etária da primeira infância, que vai de zero a seis anos; acima de dois anos de idade até os três anos, o porcentual foi de 29,6\%; de três a seis anos, de $56,1 \%$, totalizando $85,7 \%$ de todas as crianças.

Os participantes da pesquisa possuíam em média 4,45 anos ( $D P=2,45)$, com peso médio de 20,29 $\mathrm{Kg}(\mathrm{DP}=8,56)$ e 1,08 metros de altura em média $(\mathrm{DP}=0,20)$, como mostra a tabela 1.

Tabela 1 - Descrição da idade, peso e Altura dos participantes.

\begin{tabular}{lccc}
\hline & Idade & Peso & Altura \\
\hline Média & 4,45 & 20,29 & 1,08 \\
Desvio padrão (DP) & 2,45 & 8,56 & 0,20 \\
Mediana & 4,00 & 18,35 & 1,07 \\
Mínimo & 2 & 6,2 &, 71 \\
Máximo & 11 & 49,6 & 1,93 \\
\hline
\end{tabular}

Fonte: Autoria própria.

A tabela 2 mostra que 12,2\% das crianças estavam com sobrepeso e que $25,5 \%$ com obesidade. Ou seja, um total de $37,7 \%$ estavam entre sobrepeso e obesidade.

No Brasil, na faixa etária pediátrica, a prevalência de excesso de peso se encontra entre 10,8\% a 33,8\%. A partir dos cinco anos de idade, é notório que o excesso de peso e a obesidade são bastante prevalentes em todas as regiões brasileiras e em todas as classes econômicas. Vale salientar que o número de crianças acima do peso aumentou mais que o dobro entre 1989 e 2009, passando 
de $15 \%$ para $34,8 \%$. Já os índices de obesidade cresceram mais de $300 \%$ nesse mesmo grupo etário, indo de 4,1\% em 1989 para 16,6\% em 2008- 2009 (SBP, 2012).

Tabela 2 - Descrição da prevalência de obesidade na amostra.

\begin{tabular}{lcc}
\hline & $F$ & $\%$ \\
\hline$<$ Percentil 3 - Magreza & 5 & 5,1 \\
$>$ ou $=$ Percentil 3 e < Percentil 85 - Eutrófica & 56 & 57,1 \\
$>$ ou $=$ Percentil 85 e < Percentil 97 - Sobrepeso & 12 & 12,2 \\
$>$ ou $=$ Percentil 97 e < ou = Percentil 99,9 - Obesidade & 25 & 25,5 \\
\hline
\end{tabular}

Fonte: Autoria própria.

A prevalência de excesso de peso (obesidade e sobrepeso) foi de 37,2\% para o sexo masculino e $38,4 \%$ para o sexo feminino. Em um estudo realizado por Simon et al. (2009), observou-se que essa prevalência foi de $37,2 \%$ para o sexo masculino e $33,4 \%$ para o sexo feminino. Segundo dados da Pesquisa de Orçamentos Familiares (POF), realizada em 2008 e 2009, o Brasil apresenta uma taxa de aproximadamente $32 \%$ das crianças e adolescentes do sexo feminino e mais de $34 \%$ do sexo masculino com sobrepeso ou obesidade, sendo $15 \%$ classificados como obesos (NUNES et al., 2015).

Em um estudo feito na rede pública de ensino da cidade de Formiga - MG, realizado por Fernandes, Penha e Braga (2012), foi encontrado um total de 17,92\% de crianças com sobrepeso e obesidade, mostrando uma diferença em relação aos números encontrados nesse estudo.

Proporcionalmente, grande parte das mães e dos pais das crianças não finalizou o ensino fundamental e possuía idades entre 25 e 33 anos, como está descrito na tabela 3.

Entre as crianças com sobrepeso e obesidade, 32,4\% das mães ainda não tinham concluído o ensino fundamental. Schuch et al. (2013), em um estudo realizado com pré-escolares nos estados do Rio Grande do Sul e de Santa Catarina, demonstraram que, entre as variáveis associadas a uma maior prevalência de excesso de peso nas crianças, destacou-se o grau de escolaridade materna, em que 
os filhos de mães com ensino superior tinham menores prevalências de excesso de peso.

Os comportamentos alimentares das crianças são bem estabelecidos durante a primeira infância. Alguns fatores como aleitamento materno, início do uso da fórmula e introdução dos sólidos possivelmente se relacionam com as chances de desenvolver obesidade infantil. Dessa forma, é importante ressaltar que os pais são importantes modelos para a construção dos comportamentos alimentares de seus filhos. As crianças de baixo nível socioeconômico têm maiores taxas de obesidade, tornando a intervenção precoce particularmente importante (WILSON et al., 2015).

Parra e Mota (2012), em seus artigos de revisão sobre causas e consequências relacionadas à obesidade infantil, destacam que o relacionamento intrafamiliar e a baixa escolaridade materna podem ser considerados como causas socioculturais de excesso de peso na infância.

Indicadores socioeconômicos influenciam a acessibilidade aos recursos que podem afetar medidas comportamentais, tais como dieta e atividade física, relevantes para o desenvolvimento de dislipidemia, entre outros fatores de risco de doenças cardiovasculares. Na população adulta, indicadores socioeconômicos como a educação e o nível de renda foram mostrados como tendo impacto cardiovascular e fatores de risco para doenças, incluindo os níveis de lipídios. Dentro de populações pediátricas, alguns estudos têm identificado uma relação inversa entre indicadores socioeconômicos, tais como renda familiar, enquanto outros não encontraram quaisquer relações (MARTINEZ et al., 2016). 
Tabela 3 - Descrição da escolaridade e da idade de mães e pais.

\begin{tabular}{lcccc}
\hline & \multicolumn{2}{c}{ Mãe } & \multicolumn{2}{c}{ Pai } \\
& $\mathrm{F}$ & $\%$ & $\mathrm{~F}$ & $\%$ \\
\hline Escolaridade & 0 & 0,0 & 9 & 9,2 \\
Sem escolaridade & 37 & 37,8 & 46 & 46,9 \\
Ensino fundamental incompleto & 16 & 16,3 & 9 & 9,2 \\
Ensino fundamental completo & 10 & 10,2 & 10 & 10,2 \\
Ensino médio incompleto & 30 & 30,6 & 23 & 23,5 \\
Ensino médio completo & 2 & 2,0 & 0 & 0,0 \\
Ensino superior incompleto & 3 & 3,1 & 1 & 1,0 \\
Ensino superior completo & & & & \\
Idade & 22 & 22,4 & 17 & 17,3 \\
Abaixo de 25 anos & 44 & 44,9 & 43 & 43,9 \\
25-34 anos & 30 & 30,6 & 27 & 27,6 \\
35-45anos & 1 & 1,0 & 9 & 9,2 \\
46-55 anos & 1 & 1,0 & 2 & 2,0 \\
Acima de 55 anos & & & & \\
\hline
\end{tabular}

Fonte: Autoria própria.

A tabela 4 faz uma comparação entre o perfil de obesidade e dados demográficos de natureza contínua (ordinal). Encontrou-se resultado estatisticamente significativo apenas para o peso, confirmando que as crianças com sobrepeso e obesidade possuíam maior mediana de peso (mediana $=19,8$ ).

Barbosa et al. (2016), em seu estudo, pesquisaram a prevalência e os fatores sociodemográficos relacionados aos indicadores antropométricos que estão aumentados nas crianças. Foi observado que a presença de pelo menos um indicador antropométrico encontrava-se aumentado em 16,9\% das crianças. 
Tabela 4 - Comparação das medidas demográficas entre o perfil de obesidade.

\begin{tabular}{|c|c|c|c|c|c|c|c|}
\hline & \multicolumn{2}{|c|}{ Magreza } & \multicolumn{2}{|c|}{ Eutrófica } & \multicolumn{2}{|c|}{ Sobrepeso - obesidade } & \multirow[b]{2}{*}{ Sig. } \\
\hline & Média (DP) & Mediana & Média (DP) & Mediana & Média (DP) & Mediana & \\
\hline Idade & $5,4(3,43)$ & 4 & $4,55(2,24)$ & 5 & $4,16(2,64)$ & 3 & 0,51 \\
\hline Peso & $14,32(7,39)$ & 13 & $18,48(5,93)$ & 17,95 & $\begin{array}{c}23,85 \\
(10,70)\end{array}$ & 19,8 & $<0,01$ \\
\hline Altura & $1,23(0,45)$ & 1,07 & $1,08(0,16)$ & 1,10 & $1,06(0,19)$ & 1,01 & 0,21 \\
\hline $\begin{array}{l}\text { Renda da sua } \\
\text { família }\end{array}$ & $1,60(0,54)$ & 2 & $1,5(0,53)$ & 1 & $1,51(0,50)$ & 2 & 0,92 \\
\hline $\begin{array}{l}\text { Escolaridade } \\
\text { da sua mãe }\end{array}$ & $3,20(1,30)$ & 3 & $3,39(1,44)$ & 3 & $3,76(1,49)$ & 4 & 0,44 \\
\hline $\begin{array}{l}\text { Escolaridade } \\
\text { do seu pai }\end{array}$ & $3,0(1)$ & 3 & $3,0(1,47)$ & 2 & $2,89(1,44)$ & 2 & 0,94 \\
\hline $\begin{array}{l}\text { Idade da sua } \\
\text { mãe }\end{array}$ & $2,0(0,70)$ & 2 & $2,07(0,73)$ & 2 & $2,24(0,92)$ & 2 & 0,57 \\
\hline $\begin{array}{l}\text { Idade do seu } \\
\text { pai }\end{array}$ & $2,4(0,89)$ & 2 & $2,36(0,90)$ & 2 & $2,32(1,02)$ & 2 & 0,98 \\
\hline
\end{tabular}

Fonte: Autoria própria.

As crianças que se encontram entre sete e nove anos relacionam-se com um aumento de chance de desenvolver obesidade. Assim, torna-se muito preocupante o aumento da prevalência de sobrepeso e obesidade nessa faixa etária (SBP, 2012). No presente estudo, $57,1 \%$ das crianças dentro dessa faixa etária encontravam-se com sobrepeso ou obesidade, porém é importante destacar que o total de crianças entrevistadas entre sete e nove anos foi muito baixo.

A tabela 5 mostra que, proporcionalmente, menos pessoas do sexo masculino são magras e mais pessoas com casos de obesidade na família possuem sobrepeso/obesidade, em que $52,3 \%$ das crianças com sobrepeso e obesidade tinham alguma pessoa também obesa na sua família. Tais associações apresentaram resultados estatisticamente significativos. 
Tabela 5 - Associação entre perfil de obesidade, sexo, obesidade na família e local que estuda.

\begin{tabular}{|c|c|c|c|c|}
\hline & \multicolumn{3}{|c|}{ IMC } & \multirow[t]{2}{*}{ Sig. } \\
\hline & Magreza & Eutrófica & Sobrepeso/obeso & \\
\hline \multicolumn{5}{|l|}{ Sexo } \\
\hline Feminino & $5(12,8 \%)$ & $19(48,7 \%)$ & 15 (38,5\%) & \multirow[t]{2}{*}{0,01} \\
\hline Masculino & $0(0,0 \%)$ & $37(62,7 \%)$ & $22(37,3 \%)$ & \\
\hline \multicolumn{5}{|c|}{ Obesidade na família } \\
\hline Sim & $1(2,3 \%)$ & 20 (45,5\%) & 23 (52,3\%) & \multirow[t]{2}{*}{0,02} \\
\hline Não & $4(7,4 \%)$ & $36(66,7 \%)$ & $14(25,9 \%)$ & \\
\hline \multicolumn{5}{|l|}{ Onde estuda } \\
\hline Escola pública & $3(10,3 \%)$ & $18(62,1 \%)$ & $8(27,6 \%)$ & \multirow[t]{3}{*}{0,19} \\
\hline Escola privada & $0(0,0 \%)$ & $14(70,0 \%)$ & $6(30,0 \%)$ & \\
\hline Não estudo & $2(4,1 \%)$ & $24(49,0 \%)$ & $23(46,9 \%)$ & \\
\hline
\end{tabular}

Fonte: Autoria própria.

Foi observado que 29,6\% das crianças estudavam em escola pública e 20,4\% em escola particular. Entre as crianças com obesidade e sobrepeso, 21,6\% estudavam em escola pública e 16,2\% em escolar particular e o restante ainda não estava estudando. De acordo com Foltz et al. (2015), o comportamento de saúde das crianças é influenciado por fatores ambientais, e, entre esses, incluem-se as formas de educação da criança tanto no ambiente familiar quanto no ambiente escolar. Portanto, abordar a obesidade infantil requer prevenção e intervenções de tratamento em todas as configurações, programas e sistemas.

Em um estudo comparando a prevalência de obesidade entre crianças de escolas públicas e particulares, Miranda et al. (2015) demonstraram que a prevalência de sobrepeso e obesidade infantil na escola privada é maior quando comparado aos da escola pública. Outro dado encontrado nesse estudo foi que 0 percentual mais alto de gordura corporal foi encontrado nos estudantes da instituição privada. Dessa forma, nota-se que esses resultados não são de acordo com os dados encontrados no presente estudo. 


\section{CONCLUSÃO}

Por meio da análise dos dados, pode-se notar uma alta prevalência de crianças que frequentam a Estratégia Saúde da Família com obesidade e sobrepeso. Um total de $12,2 \%$ das crianças estava com sobrepeso e $25,5 \%$ com obesidade, representando que $37,7 \%$ das crianças da amostra eram obesas ou tinham sobrepeso. Isso é refletido no atual padrão da sociedade em que há um crescimento nas taxas de crianças com excesso de peso.

A amostra teve predomínio de crianças na faixa etária entre dois e seis anos e do sexo masculino. Foi alcançado um total de 98 crianças entrevistadas, pois a maioria das Unidades Básicas de Saúde trabalha com a faixa etária abaixo de dois anos e alguns pais decidiram não aceitar que seus filhos participassem do estudo.

Através da análise e observação dos dados colhidos, foi possível caracterizar os participantes da pesquisa quanto a variáveis como idade, peso, altura e IMC. Também foram observadas as características demográficas como idade dos pais, escolaridades materna e paterna, encontrando relação de excesso de peso em filhos de mães com baixa escolaridade.

Entre as limitações para a coleta dos dados, notou-se que existem Unidades Básicas de Saúde na cidade que só realizam campanhas para obtenção de dados antropométricos de crianças abaixo de dois anos, as quais não foram incluídas na pesquisa.

Quanto aos benefícios desse estudo, espera-se alertar a população sobre o aumento da prevalência de obesidade infantil e quais são os dados sociodemográficos mais relacionados a essa doença. Dessa forma, percebe-se que é necessário esclarecer as pessoas a respeito desse tema e suas possíveis consequências para a saúde. 


\section{REFERÊNCIAS BIBLIOGRÁFICAS}

ABESO. Associação Brasileira para o Estudo da Obesidade e da Síndrome Metabólica. Disponível em: <www.abeso.org.br/atitude-saudavel/mapa-obesidade>. Acesso em: 28 ago. 2016.

BARBOSA FILHO, V.C; CORDEIRO, Valter; FAGUNDES, R. R.; LOPES, A. S.; SOUZA, E. A. Presença isolada e combinada de indicadores antropométricos elevados em crianças: prevalência e fatores sociodemográficos associados. Ciência Saúde Coletiva, v. 21, n. 1, p. 213-224, 2014.

FOLTZ, J. L.; BELAY, B.; DOOYEMA, C. A; et al. Childhood Obesity Research Demonstration (CORD): The Cross-Site Overview and Opportunities for Interventions Addressing Obesity Community-Wide. Child Obes, v. 11, n.1, Feb. 2015. Disponível em: <https://www.ncbi.nlm.nih.gov/pubmed/25679059>. Acesso em: 8 out. 2016.

JORTBERG, B. T.; ROSEN, R.; ROTH, S.; et al. The Fit Family Challenge: a primary care childhood obesity pilot intervention. JABFM, v. 29, n. 4, p. 434-443, Jul./Aug 2016. Disponível em: <https://www.ncbi.nlm.nih.gov/pubmed/27390374>. Acesso em: 10 out. 2016.

KUHLE, S.; DOUCETTE, R.; KIRK, S. F. L.; et al. Successful childhood obesity management in primary care in Canada: what are the odds? Peer J, v. 3, p. 1-17, Oct. 2015. Disponível em: https://www.ncbi.nlm.nih.gov/pubmed/26623175. Acesso em: 2 set. 2016.

LEAL, V. S.; LIRA, P. I. C.; OLIVEIRA, J. S.; et al. Excesso de peso em crianças e adolescentes no estado de Pernambuco, Brasil: prevalência e determinantes. Cad. Saúde Pública, v. 28, n. 6, p. 1175-1182, jun. 2012.

MARTINEZ, E. E.; FORBES, P. W.; O'BRIEN, S. E.; et al. Census tract based income level and lipid levels in urban pediatric primary care: a retrospective study. BMC Pediatrics, v. 16, jul. 2016. Disponível em: <https://www.ncbi.nlm.nih.gov/pubmed/27391043>. Acesso em: 12 set. 2016.

MIZIARA, Angela Maria Borges; VECTORE, Celia. Excesso de peso em escolares: percepções e intercorrências na escola. Psicol. Esc. Educ, v. 18, n. 2, p. 283-291, mai./ago. 2014.

RESNICOW, K.; McMASTER, F.; BOCIAN, A.; et al. Motivational Interviewing and Dietary Counseling for Obesity in Primary Care: An RCT. Pediatrics, v. 135, n. 4, p. 649-657, Apr. 2015. Disponível em: <https://www.ncbi.nlm.nih.gov/pubmed/25825539>. Acesso em: 12 set. 2016.

RODRIGUES, A. M.; ALVES, O. M. A; AMORIM, E. C. B. L. Freitas. Impacto do Projeto de Intervenção na Obesidade Infantil no primeiro ciclo de um agrupamento de escolas. Rev. Enf. Ref., v. 4, n.5, p. 57-64, abr./jun. 2015.

SCHUCH, I. CASTRO, T. G. de; VASCONCELOS, F. A. G. de; et al. Excesso de peso em crianças de pré-escolas: prevalência e fatores associados. J. Pediatr., v. 89, n. 2, p. 179-188, 2015. Disponível em: <http://www.scielo.br/scielo.php?script=sci_arttext\&pid=S00215572013000200012>. Acesso em: 28 ago. 2016.

SERRA-PAYA, N.; ENSENYAT, A.; CASTRO-VIÑUALES, I.; et al. Effectiveness of a Multi Component Intervention for Overweight and Obese Children (Nereu Program): A Randomized Controlled Trial. PLoS One, vol. 10, n. 12, p. 1-14, dec. 2015. Disponível em: <https://www.ncbi.nlm.nih.gov/pubmed/26658988>. Acesso em: 3 set. 2016. 
SILBERBERG, M.; CARTER-EDWARDS, L.; MURPHY, G.; et al. Treating Pediatric Obesity in the Primary Care Setting to Prevent Chronic Disease: Perceptions and Knowledge of Providers and Staff. N C Med J, v. 73, n. 1, p. 9-14, jun. 2012. Disponível em: <https://www.ncbi.nlm.nih.gov/pubmed/22619846>. Acesso em: 10 out. 2016.

SIMON, V. G. N.; SOUZA, J. M. P. de; LEONE, C.; et al. Prevalência de sobrepeso e obesidade em crianças de dois a seis anos matriculadas em escolas particulares no município de São Paulo. Rev. Bras. Crescimento Desenvolv., v. 19, n. 2, p. 211-218, 2009. Disponível em:<http://pepsic.bvsalud.org/scielo.php?script=sci_arttext\&pid=S010412822009000200002> Acesso em: 28 ago. 2016.

SOCIEDADE BRASILEIRA DE PEDIATRIA. Obesidade na infância e adolescência - Manual de Orientação. Departamento Científico de Nutrologia. 2ª . Ed. - São Paulo: SBP, 2012, 142 p. 